Pacific Journal of Mathematic 


\title{
A GALOIS THEORY FOR LINEAR TOPOLOGICAL RINGS
}

\author{
B. L. ELKINS
}

Separable algebras have been studied recently by $M$. Auslander, D. Buchsbaum and Chase-Harrison-Rosenberg. The question of a Galois theory for linear topological rings opposite to the Krull type theory obtained in the above works was raised by $H$. Röhrl. In this paper, a Galois theory relating the complete subalgebras of restricted type of a complete algebra $A$ to a set of subgroups of a discrete group $G$ of automorphisms of $A$ is developed.

The notion of a linear topological module has been discussed in [1], [5], [6], [7]; while the concepts pertaining to separables algebras are now available in the monograph [4] for the most part. We employ two results of [3] which we will state below. All rings considered will be commutative with 1 .

Definition 0.1 [3]. Two ring morphisms $A \stackrel{f}{\longrightarrow} B$ are strongly distinct if, for each nonzero idempotent $e \in B$, there is $a \in A$ with $f(a) e \neq g(a) e$. Where $B$ is connected, $f$ and $g$ are strongly distinct if and only if they are distinct.

THEOREM 0.2 [3]. Let $G$ be a finite group of automorphisms of the ring $A$ having (pointwise) fixed ring $k$. The following statements are equivalent:

(0) A is a separable k-algebra [and the elements of $G$ are pairwise strongly distinct].

(1) There are families of elements of $A,\left(x_{2}\right)_{i=1}^{n},\left(y_{i}\right)_{i=1}^{n}$ with

$$
\sum_{i=1}^{n} x_{\imath} \sigma\left(y_{i}\right)=\delta_{1 \sigma}
$$

for each $\sigma \in G$, where $\delta_{1 \sigma}$ is the Kronecker delta.

(2) For each $\sigma \in G \backslash\{1\}$ and each maximal ideal $m<A$, there is $a \in A$ with $a-\sigma(a) \notin m$.

(3) For each connected k-algebra $B$ and each pair $A \underset{g}{\stackrel{f}{\longrightarrow}} B$ of $k$-algebra morphism, there is a unique $\sigma \in G$ with $\sigma g=f$.

Proof. (0) $\rightarrow(1) \rightarrow(2) \rightarrow(0)$ is contained in [3], Theorem (1.3), and the implication (2) $\rightarrow(3)$ is Corollary (3.2) of [3]. We establish (3) $\rightarrow$ (2). Let $m<A$ be a maximal ideal and suppose $\sigma \in G \backslash\{1\}$. Then the 
$k$-algebra $A / m$ is connected, so the two $k$-algebra morphisms $q, \sigma q$ : $A \rightarrow A / m$ are distinct ( $q$ is the quotient map), otherwise $\sigma=1$. Hence, there is $a \in A$ with $a-\sigma(a) \notin m$.

DEFINITION 0.3 [3]. When any of the equivalent conditions (0)(3) of (0.2) hold for $(A, G)$, we call $(A, G)$ a Galois extension of $k$ with group $G$.

Note that when $A$ is connected and $(A, G)$ is a Galois extension of $k,(0.2)(3)$ shows that $G$ the full group of $k$-algebra automorphisms of $A$.

Definition 0.4 [3]. Let $(A, G)$ be a Galois extension of $k$ and let $B$ be a subring of $A$. $B$ will be called $G$-strong if the restrictions to $B$ of any two elements of $G$ are either equal or strongly distinct.

TheOREM 0.5 ([3] 2.3). Let $(A, G)$ be a Galois extension of $k$. Then there is Galois correspondence $(g, r)$ between the set of separable $k$-subalgebras of $A$ which are G-strong and the set of subgroups of $G$. If $B$ is a separable G-strong k-subalgebra of $A$, then $g(B):=\{\sigma \in$ $G \mid \sigma(b)=b$ for all $b \in B\}$. Moreover, if $\sigma \in G, g(\sigma B)=\sigma g(B) \sigma^{-1} . \quad A$ subgroup $H$ of $G$ is normal in $G$ if and only if $r(H):=\{a \in A \mid \sigma(a)=$ a for all $\sigma \in H\}$ is a $G$-invariant subalgebra of $A$, in which case $(r(H)$, $G / H)$ is a Galois extension of $k$ with group $G / H$.

We now pass to linear topological case.

Definition 0.6. The ring $A$ with a filter basis of ideals $\mathscr{C}(A)$ has a linear topology with $a \in A$ having a basis of neighborhoods the family $(a+U) U \in \mathscr{C}(A)$, and the pair $(A, \mathscr{C}(A))$ or briefly $A$ will be called a linear topological ring. A linear topological k-algebra is a continuous ring morphism

$$
(k, \mathscr{U}(k)) \stackrel{\rho}{\longrightarrow}(A, \mathscr{U}(A)) .
$$

1. Quasi-Galois extensions. Consider the following situation:

(0) $k \rightarrow A$ is a linear topological $k$-algebra.

(1) $F$ is a final subset of $\mathscr{U}(A)$.

(2) $I \in F$ implies that $A / I$ is a connected Galois extension of $k / k \cap I$ with Galois group $G_{I}$.

Proposition 1.1. There is a unique contravariant monic valued functor $G: F \rightarrow G r\left(G r\right.$ is the category of groups) such that $G(I)=G_{I}$, and such that $I \leqq I^{\prime}$ in $F$ implies the commutativity of the diagram: 


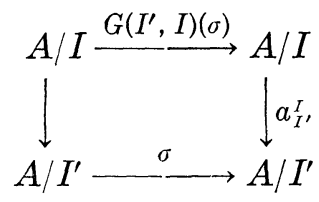

for each $\sigma \in G\left(I^{\prime}\right)$, where $a_{I^{\prime}}^{I}$ is the canonical quotient map.

Proof. For each $\sigma \in G\left(I^{\prime}\right)$, there is by (0.2), (3), a unique $\sigma^{\prime} \in G(I)$ such that $\sigma^{\prime} a_{I^{\prime}}^{I}=a_{I^{\prime}}^{I} \sigma$. We define $G\left(I^{\prime}, I\right)(\sigma):=\sigma^{\prime}$. The uniqueness available in (0.2), (3), guarantees that $G\left(I^{\prime}, I\right)$ is a group morphism, and the surjectivity of $a_{I}^{I}$, entails the injectivity of $G\left(I^{\prime}, I\right)$.

Definition 1.2. The triple $(A, F, G)$ will be called an extension of $k$ if:

(0) $k \rightarrow A$ is a linear topological $k$-algebra.

(1) $F$ is a final subset of $U(A)$; so $F$ is a filter basis.

(2) $G: F \rightarrow G r$ is a contravariart monic valued functor such that

(i) $G(I)$ is a finite subgroup of the group of $k / k \cap I$-automorphisms of $A / I$;

(ii) for each $I \leqq I^{\prime}$ in $F$ and $\sigma \in G\left(I^{\prime}\right)$ the diagram of (1.1) is commutative.

If for each $I \in F,(A / I, G(I))$ is a Galois extension of $k / k \cap I$ with Galois group $G(I)$, we will call $(A, F, G)$ a quasi-Galois extension of $k$ with group $G$.

An immediate consequence of (1.1) is the

COROLlary 1.3. If $(A, F, G)$ is a quasi-Galois extension of $k$, and if for each $I \in F, A / I$ is connected, then the functor $G$ is uniquely determined.

Let $(A, F, G)$ be an extension of $K$. We will define a group $\hat{G}$ of continuous $k$-automorphisms of $\hat{A}$

$$
\left(\hat{A}=\lim _{I \in \mathbb{\mathscr { T }}(A)} A / I \quad \text { and } \quad \mathscr{C}(\hat{A})=\left\{\operatorname{ker}\left(\hat{A} \stackrel{a_{I}}{\longrightarrow} A / I\right) I \in \mathscr{C}(A)\right\}\right)
$$

and show that when $(A, F, G)$ is a quasi-Galois extension of $k$, then there is a Galois correspondence $(g, r)$ between a specific class of subgroups of $\hat{G}$ and a class of complete $\hat{k}$-subalgebras of $\hat{A}$. Each of these classes is characterized by the quality of their approximations, i.e., we require that their approximations satisfy a specific condition for each $I \in F$.

Since $F$ is a filter basis, the family $(G(I))_{I \in F}$ of groups is cofiltered, 
and we can form the colimit $\hat{G}$ : $=\lim G(I)$, the colimit being taken over $I \in F$. We denote by $g_{I}: G(I) \rightarrow \widehat{G}$ the canonical colimit morphisms; they are injective, and for $I \leqq I^{\prime}$ in $F$ yield a commutative diagram:

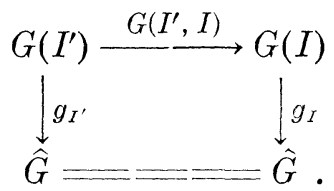

Another useful description of $\hat{G}$ is obtained as follows. Fix $I^{\prime} \in F$ and consider any $I \leqq I^{\prime}$ in $F$. We then have a commutative diagram:

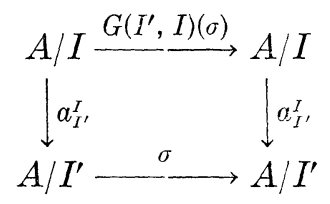

for each $\sigma \in G\left(I^{\prime}\right)$. Evidently, the family of morphism $\left(G\left(I^{\prime}, I\right)(\sigma)\right)_{I ミ I^{\prime}}$ is filtered and compatible with the quotient maps $a_{I}^{I}$, so we can form the limit $\hat{\sigma}$ of this family, obtaining, for each $I \leqq I^{\prime}$, the commutative diagram:

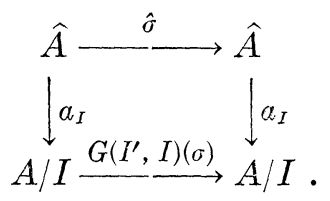

We let $H$ denote the set of all such $\hat{\sigma}$ for $I^{\prime} \in F$ and $\sigma \in G\left(I^{\prime}\right)$ arbitrary. The foregoing diagram shows that each $\hat{\sigma}$ is a continuous $\hat{k}$-automorphism of $\hat{A}$. If $\hat{\sigma}, \hat{\tau} \in H$, say $\sigma \in G\left(I^{\prime}\right)$ and $\tau \in G\left(\tau^{\prime \prime}\right)$, we define $\hat{\sigma} \hat{\tau}=\hat{\mu}$, where $\mu=G\left(I^{\prime}, I\right)(\sigma) \cdot G\left(I^{\prime \prime}, I\right)(\tau)$ and $I \leqq I^{\prime}, I^{\prime \prime}$. Since $F$ is a filter basis, $\hat{\mu}$ does not depend on $I$, and so is well-defined; moreover, this multiplication makes $H$ a group.

Proposition 1.4. The mapping $H \rightarrow \hat{G}$, given by $\hat{\sigma} \rightarrow g_{I}(\sigma)$, where $\sigma \in G(I)$, is a group isomorphism.

Proof. Define $h_{I}: G(I) \rightarrow H$ by putting $h_{I}(\sigma)=\hat{\sigma}$. The $h_{I}$ are then group morphisms compatible with the inclusions $G\left(I^{\prime}, I\right)$ for $I \leqq I^{\prime}$; hence, there is a unique group morphism $h: \hat{G} \rightarrow H$ such that $g_{I} h=$ $h_{I}$ for all $I \in F$. Next, define $g: H \rightarrow \hat{G}$ by putting $g(\hat{\sigma})=g_{I}(\sigma)$ if $\sigma \in G(I)$. To see that $g$ is well-defined, let $\hat{\sigma}=\hat{\tau}$, where $\sigma \in G\left(I^{\prime}\right)$ and $\tau \in G\left(I^{\prime \prime}\right)$, and choose $I \leqq I^{\prime}, I^{\prime \prime}$. Then 


$$
\begin{aligned}
1 & =\hat{\sigma}(\hat{\tau})^{-1}=\left[G\left(I^{\prime}, I\right)(\sigma)\right]^{\wedge} \cdot\left[G\left(I^{\prime \prime}, I\right)\left(\tau^{-1}\right)\right]^{\wedge} \\
& =\left[G\left(I^{\prime}, I\right)(\sigma) G\left(I^{\prime \prime}, I\right)\left(\tau^{-1}\right)\right]^{\wedge}
\end{aligned}
$$

This shows that the diagram:

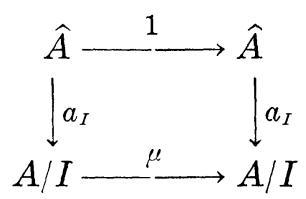

is commutative, where $\mu=G\left(I^{\prime}, I\right)(\sigma) G\left(I^{\prime \prime}, I\right)\left(\tau^{-1}\right)$. But $a_{I}$ is surjective, so we conclude that $\mu=1$, and so $G\left(I^{\prime}, I\right)(\sigma)=G\left(I^{\prime \prime}, I\right)(\tau)$, proving that $g_{I^{\prime}}(\sigma)=g_{I}\left(G\left(I^{\prime}, I\right)(\sigma)\right)=g_{I}\left(G\left(I^{\prime \prime}, I\right)(\tau)\right)=g_{I^{\prime \prime}}(\tau)$ as required.

A similar argument shows that $g$ is a group morphism. Finally, let $\sigma \in G(I)$, then $h(g(\hat{\sigma}))=h\left(g_{I}(\sigma)\right)=h_{I}(\sigma)=\hat{\sigma}$. On the other hand, each element $x$ of $\hat{G}$ has the form $g_{I}(\sigma)$ for some $I \in F$, since $F$ is a filter basis. It follows that $g(h(x))=g h\left(g_{I}(\sigma)\right)=g\left(h_{I}(\sigma)\right)=g(\hat{\sigma})=$ $g_{I}(\sigma)=x$. Thus, we have the group identities $1=g h$ and $1=h g$ showing that $g$ is a group isomorphism.

Proposition 1.5. If $(A, F, G)$ is an extension of $k$ such that for each $I \in F$, the fixed ring of $G(I)$ is $k / k \cap I$, then the fixed ring of $\hat{G}$ is $\hat{k}$.

Proof. We have already observed that $G(I) \leqq \operatorname{Auto}_{k \mid k \cap I}(A / I)$ implies that the elements of $\hat{G}$ are $\hat{k}$-automorphisms of $\hat{A}$. Now suppose $\alpha \in \hat{A}$ belongs to the fixed ring of $\hat{G}$. Then we have commutative diagram:

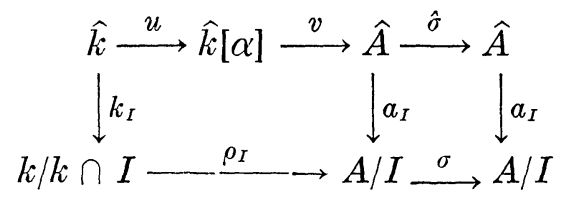

where $\rho_{I}, u$ and $v$ are the canonical inclusions and $u v=\hat{\rho}: \hat{k} \rightarrow \hat{A}$ is the limit of the morphisms $\rho_{I}$, and where $\sigma \in G(I) . \hat{k}[\alpha]$ has the topology induced by $v$, so all the morphisms are continuous. By hypothesis, $v a_{I} \sigma=v \hat{\sigma} a_{I}=v a_{I}$, so that $v a_{I}$ factors through the fixed ring of $G(I)$, namely $k / k \cap I$. Let the factorization be $v a_{I}=w_{I} \rho_{I}$. For $I \leqq I^{\prime}$ in $F$, we have $w_{I} k_{I^{\prime}}^{I} \rho_{I^{\prime}}=w_{I} \rho_{I} a_{I^{\prime}}^{I}=v a_{I} a_{I^{\prime}}^{I}=v a_{I^{\prime}}=w_{I^{\prime}} \rho_{I^{\prime}}$ and since $\rho_{I^{\prime}}$ is monic, $w_{I} k_{I^{\prime}}^{I}=w_{I^{\prime}}$. Thus, we obtain a family $\left(w_{I}\right) I \in F$ compatible with the morphisms $k_{I}^{I}: k / k \cap I \rightarrow k / k \cap I^{\prime}$. Passing to the limit, we obtain a commutative diagram 


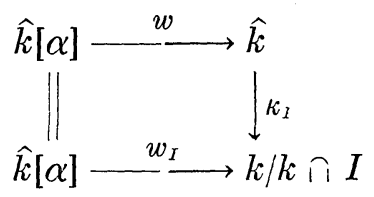

for each $I \in F . \quad w$ is continuous, and $v a_{I}=w_{I} \rho_{I}=w k_{I} \rho_{I}=w(u v) a_{I}$ for each $I \in F$, so passing to the limit again, $v=(w u) v$. But $v$ is monic, so we conclude that $1=w u$ showing that $u$ is surjective. Since $u$ is already injective, $u$ is an isomorphism and we conclude that $\alpha \in \hat{k}$ as desired.

THEOREM 1.6. Let $(A, F, G)$ be an extension of $k$ such that for each $I \in F$, the fixed ring of $G(I)$ is $k / k \cap I$. Then the following statements are equivalent.

(0) $(A, F, G)$ is a quasi-Galois extension of $k$.

(1) For each $\hat{\sigma} \in \hat{G} \backslash 1$ and each open, maximal ideal $m<\hat{A}$, there is $x \in \hat{A}$ with $x-\hat{\sigma}(x) \notin m$.

In addition, if $I \in F$ implies that $A / I$ is connected, (0) and (1) are equivalent to a third condition.

(2) $A$ is a quasi-separable k-algebra, i.e., $I \in F$ implies $A / I$ is a separable $k / k \cap I$ - algebra.

Proof. Consider the diagram

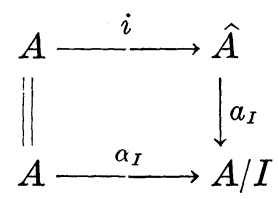

where $i$ is the canonical limit morphism, and $\alpha_{I}$ and $\alpha_{I}$ are the quotient maps. Let $m<\hat{A}$ be an open, maximal ideal and let $\hat{\sigma} \in \hat{G} \backslash 1$. We may suppose $I \in F$ is such that $m \geqq \operatorname{ker}\left(a_{I}\right)$ and $\hat{\sigma}=g_{I}(\sigma)$. Since $i^{-1}(m)$ is an open, maximal ideal of $A, \alpha_{I}\left(i^{-1}(m)\right)$ is a maximal ideal of $A / I$, and $\sigma \in G \backslash 1$ shows that there is $a \in A / I$ such that $a-\sigma(a) \notin \alpha_{I}\left(i^{-1}(m)\right)$, assuming (0), by (0.2). Suppose $y \in A$ is such that $\alpha_{I}(y)=a$, then $i(y)-\hat{\sigma} i(y) \notin m$; otherwise, $a_{I} i(y)-a_{I} \hat{\sigma} i(y)=\alpha_{I}(y)-\sigma \alpha_{I}(y) \in a_{I}(m)=$ $\alpha_{I}\left(i^{-1}(m)\right)$ contrary to our choice of $\alpha_{I}(y)=a$. Thus, $i(y)-\hat{\sigma} i(y) \notin m$ as desired.

Now suppose $m$ is a maximal ideal of $A / I$ and let $\sigma \in G(I) \backslash 1$. Then $a_{I}^{-1}(m)$ is an open, maximal ideal of $\hat{A}$, and $g_{I}(\sigma)=\hat{\sigma} \in \hat{G} \backslash 1$. We obtain, therefore, $x \in \hat{A}$ with $x-\hat{\sigma}(x) \notin a_{I}^{-1}(m)$. It follows that $a_{I}(x)-a_{I} \hat{\sigma}(x)=$ $a_{I}(x)-\sigma a_{I}(x) \notin m$ showing that $A / I$ is a Galois extension of $k / k \cap I$ with Galois group $G(I)$ by (0.2).

If, in addition, $I \in F$ implies that $A / I$ is connected, and (0) holds, then by definition $A$ is a quasi-separable $k$-algebra. The converse implication follows from (0.2). 
Corollary 1.7. Suppose $(A, F, G)$ is an extension of $k$ such that for each $I \in F$, the fixed ring of $G(I)$ is $k / k \cap I$. If the condition (*) below holds, then $(A, F, G)$ is a quasi-Galois extension of $k$.

(*) For each $\hat{k}$-algebra $B$ and each pair of continuous $\hat{k}$-algebra morphisms $f, g: \hat{A} \rightarrow B$, there is a unique $\hat{\sigma} \in \hat{G}$ such that $\hat{g}=\hat{\sigma} f$.

Proof. Let $\hat{\sigma} \in \hat{G} \mid 1$ and let $m<\hat{A}$ be an open, maximal ideal. If $a-\hat{\sigma}(a) \in m$ for all $a \in A$, then the two $\hat{k}$-algebra morphisms $q: \hat{A} \rightarrow \hat{A} / m$ and $\hat{\sigma} q$ agree on $\hat{A}$, so by $(*)$ we must have that $\hat{\sigma}=1$ which is a contradiction. We conclude that there is $a \in \hat{A}$ with $a-\hat{\sigma}(a) \notin m$, and so by (1.6) $(A, F, G)$ is a quasi-Galois extension of $k$.

Definition 1.8. Let $(A, F, G)$ be an extension of $k$. For each subgroup $H$ of $\hat{G}$ let $r(H)$ denote the pointwise fixed ring of $H$ and let $H_{I}:=g_{I}^{-1}(H)$. For each $\hat{k}$-subalgebra $B$ of $\hat{A}$ let $g(B)$ denote the subgroup of $\hat{G}$ fixing $B$ elementwise.

For $I \leqq I^{\prime}$ in $F$ we then have a commutative diagram:

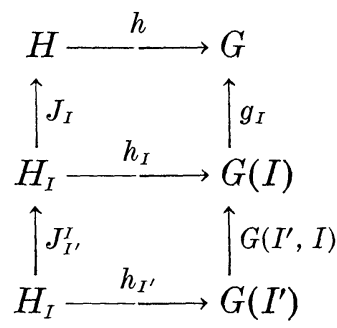

where $h, h_{I}$, and $h_{I^{\prime}}$ are the canonical inclusions, and $J_{I}$ and $J_{I^{\prime}}^{I}$ are the monomorphisms induced by $g_{I}$ and $G\left(I^{\prime}, I\right)$ respectively.

Proposition 1.9. The colimit of the family $\left(H_{I}, J_{I^{\prime}}^{I}\right)$ is $H$ with the colimit morphisms being the $J_{I}$.

Proof. We have just observed the compatibility of the family of morphisms $J_{I}$ with the mappings $J_{I^{\prime}}^{I}$, for $I \leqq I^{\prime}$ in $F$, and it remains to establish their universality. Let $x_{I}: H_{I} \rightarrow X$ be any family of group morphisms compatible with the mappings $J_{I^{\prime}}^{I}\left(I \leqq I^{\prime}\right.$ in $\left.F\right)$. Define $x: H \rightarrow X$ by putting $x(\hat{\sigma}):=x_{I}(\sigma)$, if $g_{I}(\sigma)=\hat{\sigma}$. If $g_{I^{\prime}}\left(\sigma^{\prime}\right)=\hat{\sigma}$ also, choose $I^{\prime \prime} \leqq I, I^{\prime}$ so that $J_{I}^{I^{\prime \prime}}(\sigma)=J_{I^{\prime}}^{I^{\prime \prime}}\left(\sigma^{\prime}\right)$. Then $x_{I}(\sigma)=x_{I^{\prime \prime}}\left(J_{I}^{I^{\prime \prime}}(\sigma)\right)=$ $x_{I^{\prime \prime}}\left(J_{I^{\prime}}^{I^{\prime \prime}}\left(\sigma^{\prime}\right)\right)=x_{I^{\prime}}\left(\sigma^{\prime}\right)$ shows that $x$ is a group morphism, and the equality $J_{I} x=x_{I}$ for $I \in F$ shows that $x$ is uniquely determined. Hence, $J_{I}: H_{I} \rightarrow$ $H$ is a colimit for $\left(H_{I}, J_{I}^{I}\right)$.

Next, let $H$ be a subgroup of $G$, and obtain the diagram: 


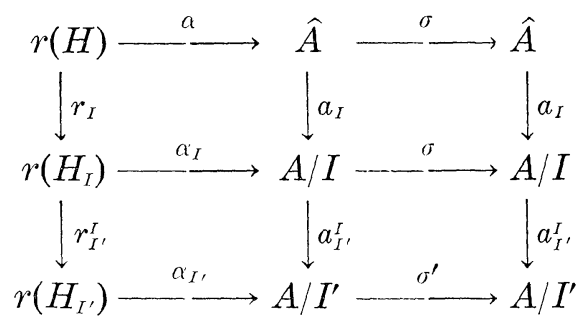

which is commutative, where $\alpha, \alpha_{I}, \alpha_{I^{\prime}}$ are inclusions providing their respective domains with the induced topology. For each $\sigma \in H_{I}, \alpha a_{I} \sigma=$ $\alpha \hat{\sigma} \alpha_{I}=\alpha \alpha_{I}$, so that $\alpha_{I}$ factors through $r\left(H_{I}\right)$, defining $r_{I}$. Then $\alpha a_{I}=$ $r_{I} \alpha_{I}$ for all $I \in F$. Similarly, if $I \leqq I^{\prime}$ in $F$, and $\sigma^{\prime} \in G\left(I^{\prime}\right)$ and $\sigma=$ $G\left(I^{\prime}, I\right)\left(\sigma^{\prime}\right)$, then $\sigma_{I} \alpha_{I^{\prime}}^{I} \sigma^{1}=\alpha_{I}^{\prime} a_{I^{\prime}}^{I}$, so that $\alpha_{I^{\prime}}^{I}$ factors through $r\left(H_{I^{\prime}}\right)$, defining $r_{I^{\prime}}^{I}$. Then $r_{I}^{I}, \alpha_{I^{\prime}}=\alpha_{I} \alpha_{I^{\prime}}^{I}$. Still using the above diagram, we obtain from the equality $r_{I^{\prime}} \alpha_{I^{\prime}}=r_{I} r_{I^{\prime}}^{I} \alpha_{I^{\prime}}$ the relation $r_{I^{\prime}}=r_{I} r_{I^{\prime}}^{I}$, since $\alpha_{I}$, is monic. This shows that the mapping $r_{I}: r(H) \rightarrow r\left(H_{I}\right)$ are compatible with the mapping $\left(r_{I^{\prime}}^{I}\right) I \leqq I^{\prime}$ in $F$.

Proposition 1.10. The mappings $r_{I}: r(H) \rightarrow r\left(H_{I}\right)$ form a limit for the family $\left(r\left(H_{I}\right), r_{I^{\prime}}^{I}\right)$.

Proof. Let $x_{I}: X \rightarrow r\left(H_{I}\right)$ be any family of continuous ring morphisms compatible with the $r_{I^{\prime}}^{r}$. Composing this family coordinatewise with the family $\left(\alpha_{I}\right) I \in F$, we obtain a family $\left(x_{I} \alpha_{I}\right) I \in F$ compatible with the canonical quotient maps $a_{I^{I}}^{I}$. Hence, there is a unique continuous mapping $x: X \rightarrow \widehat{A}$ such that $x a_{I}=x_{I} \alpha_{I}$ for each $I \in F$. Now let $\hat{\sigma} \in H$, say $\hat{\sigma}=g_{I^{\prime}}(\sigma)$ for some $I^{\prime} \in F$. For all $I \leqq I^{\prime}$ in $F, x \hat{\sigma} a_{I}=$ $x a_{I} G\left(I^{\prime}, I\right)(\sigma)=x_{I} \alpha_{I} G\left(I^{\prime}, I\right)(\sigma)=x_{I} \alpha_{I}=x \alpha_{I}$ since $G\left(I^{\prime}, I\right)(\sigma) \in H_{I}$. This being true for all small $I \in F$, passing to the limit, we have $x \hat{\sigma}=x$, showing that $x$ must factor through $r(H)$. Let $x=y \alpha$ for some $y: X \rightarrow r(H) . \quad y$ is then unique, since $\alpha$ is monic, and $y r_{I} \alpha_{I}=y \alpha a_{I}=$ $x_{I} \alpha_{I}$ implies that $y r_{I}=x_{I}$ since $\alpha_{I}$ is monic. This completes the proof.

REMARK. The topology induced by $\alpha$ on $r(H)$ coincides with the limit topology for $\operatorname{ker}\left(r_{I}\right)=\operatorname{ker}\left(r_{I} \alpha_{I}\right)=\operatorname{ker}\left(\alpha a_{I}\right)$. For the remainder of this section we assume $(A, F, G)$ is a quasi-Galois extension of $k$.

For each subgroup $H$ of $\hat{G}$ we are led to a commutative diagram:

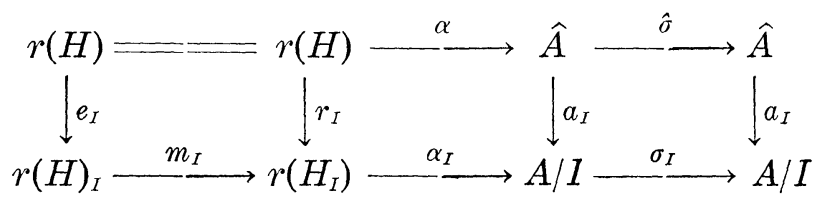


where $r(H)$ is the image of $\alpha a_{I}$ and $r(H)_{I} \leqq r\left(H_{I}\right)$, since $\sigma \in H_{I}$ implies $e_{I} \alpha_{I}^{\prime} \sigma=e_{I} \alpha_{I}^{\prime}$, where $e_{I} \alpha_{I}^{\prime}$ is the canonical factorization of $\alpha_{I}$ through $r(H)_{I}$. Since $e_{I}$ is surjective, $\alpha_{I}^{\prime} \sigma=\alpha_{I}^{\prime}$ shows that $r(H)_{I} \leqq r\left(H_{I}\right)$, say $m_{I}: r(H)_{I} \rightarrow r\left(H_{I}\right)$ so that $\alpha_{I}^{\prime}=m_{I} \alpha_{I}$. Since $\alpha_{I}$ is monic and $e_{I} m_{I} \alpha_{I}=$ $r_{I} \alpha_{I}, e_{I} m_{I}=r_{I}$, so the first square is commutative.

It follows immediately from the definitions that $H \leqq g r(H)$ for each subgroup $H$ of $\hat{G}$.

Lemma 1.11. Suppose $H \leqq \hat{G}$ satisfies the condition $I \in F \rightarrow H_{I}=$ $g\left[r(H)_{I}\right]$, where $g$ is appropriately defined. Then $g r(H)=H$.

Proof. Of course, by $g\left[r(H)_{I}\right]$ we mean the set

$$
\left\{\sigma \in G(I) \mid x \in r(H)_{I} \longrightarrow \sigma(x)=x\right\} .
$$

Let $\hat{\sigma} \in \operatorname{gr}(H)$ and suppose $g_{I}(\sigma)=\hat{\sigma}$. Then the equality $m_{I} \alpha_{I} \sigma=m_{I} \alpha_{I}$ shows that $\sigma \in g\left[r(H)_{I}\right]=H_{I}$ by hypothesis; hence $\hat{\sigma}=g_{I}(\sigma) \in H$.

Definition 1.12. Call a $\hat{k}$-subalgebra $B$ of $\hat{A} G$-strong if for each $I \in F, B_{I}$ is a $G(I)$-strong subalgebra of $A / I$.

Lemma 1.13. Let $H \leqq \widehat{G}$. The following statements are equivalent:

1.14. (0) $I \in F \rightarrow r(H)_{I}=r\left(H_{I}\right)$, i.e., $r_{I}$ is surjective.

(1) $I \in F \rightarrow H_{I}=g\left[r(H)_{I}\right]$ and $r(H)$ is a G-strong separable $\hat{k}$ subalgebra of $\hat{A}$.

Proof. Suppose (0), then since $(A, F, G)$ is a quasi-Galois extension of $k, r(H)_{I}=r\left(H_{I}\right)$ shows that $r\left(H_{I}\right)$ is a $G(I)$-strong separable $k / k \cap$ $I$-subalgebra of $A / I$ for $I \in F . \quad r(H)$ is a closed $\hat{k}$-subalgebra of the complete separated ring $\hat{A}$, i.e., is complete. Finally, $H_{I}=\operatorname{gr}\left(H_{I}\right)=$ $g\left[r(H)_{I}\right]$ by (0) and (0.5). Conversely, if (1) holds, then

$$
r\left(H_{I}\right)=r g\left[r(H)_{I}\right]=r(H)_{I}
$$

since $r(H)$ is a $G$-strong quasi-separable $\hat{k}$-subalgebra of $\hat{A}$ and $r g=$ 1 by $(0.5)$.

CoRollary 1.15. If $H \leqq \hat{G}$ satisfies (1.14), $\operatorname{gr}(H)=H$.

Now let $B$ be a complete $\hat{k}$-subalgebra of $\hat{A}$ and put $H=g(B)$. We obtain the following supplement to the last diagram

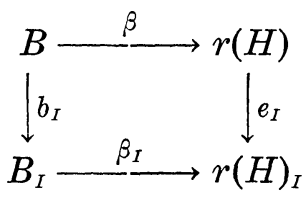


for each $I \in F$. For evidently $B \leqq r g(B)=r(H)$.

Lemma 1.16. Suppose $B$ is a complete $\hat{k}$-subalgebra of $\hat{A}$ satisfying the condition.

1.17. $I \in F \rightarrow B_{I}=r\left[g(B)_{I}\right]$.

Then $B$ is a G-strong quasi-separable $\hat{k}$-subalgebra of $\hat{A}, r g(B)=B$, and $g(B)$ satisfies Condition 1.14.

Proof. Since $B_{I}=r\left[g(B)_{I}\right]$ is the fixed ring of a subgroup of $G(I)$, it follows from (0.5) that $B_{I}$ is a $G(I)$-strong separable $k / k \cap I$-subalgebra of $A / I$, proving our first assertion. Next, we have the equalities:

$$
B=\lim _{I} B_{I}=\lim _{I}\left(r\left[g(B)_{I}\right]\right)=r\left(\underset{I}{\lim }\left[g(B)_{I}\right]\right)=r g(B)
$$

by (1.9) and (1.10). Using this fact, we obtain $[r g(B)]_{I}=B_{I}=r\left[g(B)_{I}\right]$ showing that (1.14) holds for $g(B)$.

REMARK. If $H \leqq \hat{G}$ satisfies Condition 1.14, then $r(H)$ satisfies Condition 1.17 for $r(H)_{I}=r\left(H_{I}\right)=r\left[(g r(H))_{I}\right]$ since $H=g r(H)$.

THeOREM 1.18. Let $(A, F, G)$ be a quasi-Galois extension of $k$. Then the pair of maps $(g, r)$ is a Galois correspondence between the set of all complete $\hat{k}$-subalgebras of $\hat{A}$ satisfying Condition 1.17 and the set of all subgroups of $\hat{G}$ satisfying Condition 1.14 .

Proof. We need only observe that $g r=1$ and $r g=1$ are valid equations when restricted to the sets mentioned in the statement of the theorem.

Proposition 1.19. Suppose $H$ is normal subgroup of $\hat{G}$ satisfying Condition 1.14. Then for each $I \in F, H_{I}$ is a normal subgroup of $G(I)$.

Proof. Form the diagram:

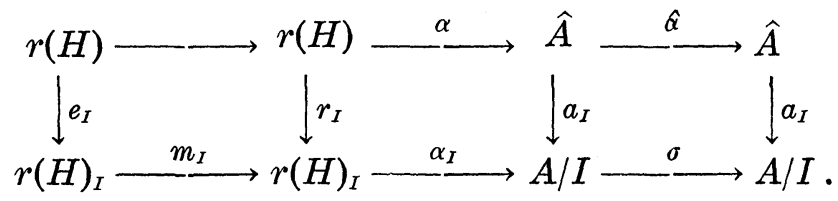

Our hypotheses on $H$ show that $r_{I}$ is surjective. Now let $\sigma \in G(I)$ and $h \in H_{I}$. Then $r_{I} \alpha_{I} \sigma^{-1} h \sigma=\alpha\left(\sigma^{-1}\right)^{\wedge} \hat{h} \hat{\sigma} a_{I}=\alpha a_{I}=r_{I} \alpha_{I}$, since

$$
\left(\sigma^{-1}\right)^{\wedge} \hat{h} \hat{\sigma} \in H \text {. }
$$


However, $r_{I}$ is surjective, so $\alpha_{I} \sigma^{-1} h \sigma=\alpha_{I}$, and we conclude that $\sigma^{-1} h \sigma \in H_{I}$ since $g r\left(H_{I}\right)=H_{I}$. Hence, $H_{I}$ is a normal subgroup of $G(I)$.

Consider the following diagram of groups:

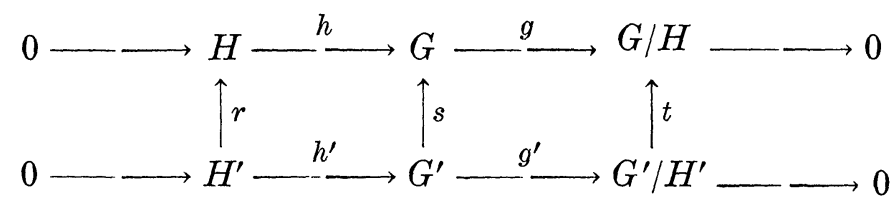

where the rows are exact, $r$ and $s$ are monomorphisms, while $t$ is the unique group morphism making the right square commutative.

Lemma 1.20. If $\left(H^{\prime}, r, h^{\prime}\right)$ is a pullback for $h$ and $s$, then $t$ is a monomorphism.

Proof. Let $t\left(x^{\prime}\right)=1$, then $g^{\prime}\left(y^{\prime}\right)=x^{\prime}$ for some $y^{\prime} \in G^{\prime}$, and so $g s\left(y^{\prime}\right)=1$. Hence $h(z)=s\left(y^{\prime}\right)$ for some $z \in H$. But since $H^{\prime}$ is a pullback, there is $z^{\prime} \in H^{\prime}$ such that $r\left(z^{\prime}\right)=z$ and $h^{\prime}\left(z^{\prime}\right)=y^{\prime}$. Therefore, $1=g^{\prime} h^{\prime}\left(z^{\prime}\right)=g^{\prime}\left(y^{\prime}\right)=x^{\prime}$, and we conclude that $t$ is a monomorphism.

Now suppose $H$ is a normal subgroup of $\hat{G}$ satisfying condition (1.14). For each $I \leqq I^{\prime}$ in $F$ we are led to a commutative diagram of groups:

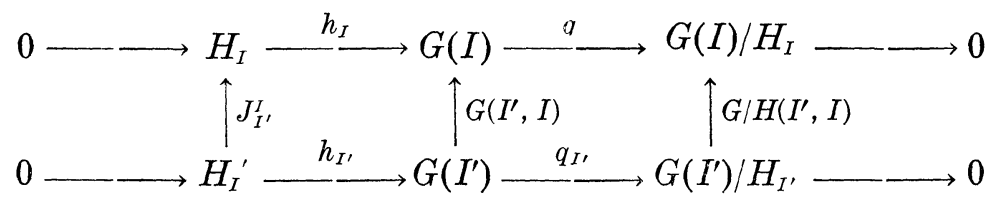

where $q_{I}$ and $q_{I^{\prime}}$ are the canonical quotient maps, and $G / H\left(I^{\prime}, I\right)$ is the map produced by the remainder making the whole diagram commutative with exact rows. Since $J_{I^{\prime}}^{I}$ and $G\left(I^{\prime}, I\right)$ are monic, while $H_{I}$, is a pullback, it follows from our foregoing Lemma that $G / H\left(I^{\prime}, I\right)$ is also a monomorphism.

Thus, we obtain a contravariant monic valued functor $G / H: F \rightarrow$ $G$ such that $I \in F$ implies that $G / H(I)=G(I) / H_{I}$ is the Galois group of $r\left(H_{I}\right)$ over $k / k \cap I$ by (0.5). Finally, the diagram

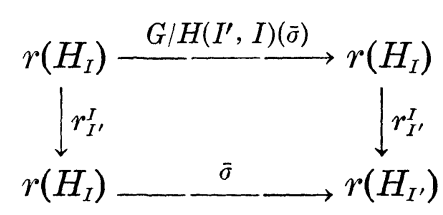

is commutative for each $\bar{\sigma} \in G / H\left(I^{\prime}\right)$. For if $\bar{\sigma}=q_{I^{\prime}}(\sigma)$, then $G / H\left(I^{\prime}\right.$, $I)(\bar{\sigma})=q_{I}\left(G\left(I^{\prime}, I\right)(\sigma)\right)$ and the corresponding diagram 


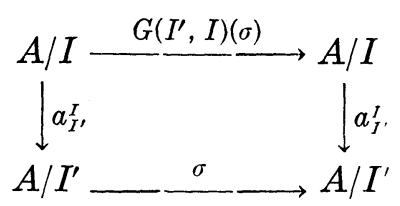

is commutative.

This establishes the corollary below.

COROLlary 1.21. Let $A$ be a separated and complete linear topological k-algebra. Suppose $(A, F, G)$ is a quasi-Galois extension of $k$, and suppose $H$ is a normal subgroup of $\hat{G}$ satisfying condition (1.14). Then there is a final subset $F^{\prime}$ of $F$ such that $\left(r(H), F^{\prime \prime} \cap r(H)\right.$, $G / H)$ is a quasi-Galois extension of $k$, where

$$
F^{\prime} \cap r(H)=\left\{I^{\prime} \cap r(H) \mid I^{\prime} \in F^{\prime}\right\} .
$$

Proof. Define $F^{\prime}$ to be the smallest subset of $F$ such that for each intersection $r(H) \cap I$ with $I \in F$, there is $I^{\prime} \in F^{\prime}$ with $r(H) \cap I^{\prime}=$ $r(H) \cap I$. Because $r(H)$ has the induced topology, $F^{\prime \prime}$ is final in $\mathscr{U}(r(H))$ and our foregoing. constructions show that $\left(r(H), F^{\prime \prime} \cap r(H), G / H\right)$ is a quasi-Galois extension of $k$.

2. Examples. In this section we will show how to construct a number of examples of the foregoing material. Two lemmata are useful in this direction.

Lemma 2.1. Let $X$ and $Y=\left(Y_{i}\right)_{i \in I}$ be distinct indeterminants over the ring $A$. Let $f \in A[X]$ be a monic polynomial, and suppose $I \leqq(A[X] /(f))[Y]$ is an ideal. Let $I^{\prime}$ be the ideal generated by the image of $I$ in $A[X, Y]$ under the canonical inclusion $A[X] /(f) \subset A[X$, $Y]$. Then we have $(A[X] /(f))[Y] / I \cong A[X, Y] /\left(f A[X, Y]+I^{\prime}\right)$.

Proof. We have a commutative diagram:

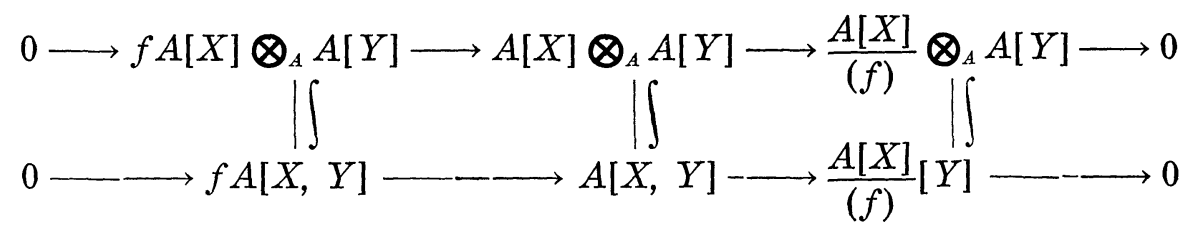

with exact rows. Hence, $\operatorname{ker}(\alpha)=f A[X, Y]$. If $\beta$ is the quotient mapping $(A[X] /(f))[Y] \rightarrow(A[X] /(f))[Y] / I$ and $\beta \alpha(P)=0$, then $\alpha(P) \in$ $I$, so there is $Q \in I^{\prime}$ such that $\alpha(P) \in I^{\prime}+f A[X, Y]$. Evidently, this latter ideal is contained in $\operatorname{ker}(\alpha \beta)$, completing the proof. 
Lemma 2.2. Suppose $I \leqq k\left[X_{1}, \cdots, X_{n}\right] \subseteq k[X], X=\left(X_{i}\right)_{i \geqq 1}$. Then $k[X] /\left([X] \cdot I+k[X] \cdot\left\langle X_{n+1}, X_{n+2}, \cdots\right\rangle\right) \cong k\left[X_{1}, \cdots, X_{n}\right] / I$.

Proof. Let $k[X] \stackrel{\Phi}{\longrightarrow} k\left[X_{1}, \cdots, X_{n}\right] \stackrel{\psi}{\longrightarrow} k\left[X_{1}, \cdots, X_{n}\right] / I$ be the composition of the evaluation at the point $\left(X_{1}, X_{2}, \cdots, X_{n}, X_{n}, 0,0, \cdots\right)$ followed by the canonical quotient morphism $\psi$. Clearly, $k[X] \cdot I+$ $k[X] \cdot\left\langle X_{n+1}, \cdots\right\rangle$ is contained in the kernel of the surjection $\Phi_{\psi}^{\prime}$; if $\psi(\Phi(f))=0$, then $f=(f-\Phi(f))+\Phi(f) \in k[X]$ shows that

$$
f \in k[X] I+k[X] \cdot\left\langle X_{n+1}, \cdots\right\rangle \text {. }
$$

1. Example of a quasi-Galois extension. Suppose $A_{0}$ is a complete Noetherian local ring with residual field $k_{0}$. Let $k_{0}<k_{1}<\cdots$ be a tower of finite Galois field extensions of $k_{0}$ with corresponding Galois groups $G\left(k_{i} / k_{0}\right)$.

Since $k_{1}$ is a finite Galois extension of $k_{0}$, we can find a monic polynomial $f_{1} \in A_{0}\left[X_{1}\right]$ such that $k_{0}\left[X_{i}\right] /\left(\bar{f}_{1}\right) \cong k_{1}$, where $\bar{f}_{1}$ is the reduction of $f_{1}$ modulo $j\left(A_{0}\right)$, the Jacobson radical of $A_{0}$. Following [8] p. 63 we see that $A_{1}=A_{0}\left[X_{1}\right] /\left(f_{1}\right)$ is a complete Noetherian local ring which is an $A_{0}$-algebra of finite type; moreover, $A_{1}$ is a Galois extension of $A_{0}$ with Galois group isomorphic to $G\left(k_{1} / k_{0}\right)$ in the sense of [3].

Since $k_{2}$ is a finite Galois extension of $k_{1}$, we repeat the above construction obtaining a monic polynomial $f_{2} \in A_{1}\left[X_{2}\right]$ such that $A_{2}$ : = $A_{1}\left[X_{2}\right] /\left(f_{2}\right)$ is a Galois extension of $A_{1}$ with Galois group $G\left(k_{2} / k_{1}\right)$.

We have the ring inclusions $A_{0} \leqq A_{0}\left[X_{1}\right] /\left(f_{1}\right) \leqq\left(A_{0}\left[X_{1}\right] /\left(f_{1}\right)\right)\left[X_{2}\right] /\left(f_{2}\right)$. Since $f_{1}$ is monic, we can view $f_{2} \in A_{0}\left[X_{1}, X_{2}\right]$ and apply Lemma $2 . ?$ to obtain the isomorphism:

$$
\frac{A_{0}\left[X_{1}\right]}{\left(f_{1}\right)}\left[X_{2}\right] /\left(f_{2}\right) \cong \frac{A_{0}\left[X_{1}, X_{2}\right]}{f_{1} A_{0}\left[X_{1}, X_{2}\right]+f_{2} A_{0}\left[X_{1}, X_{2}\right]}=\frac{A_{0}\left[X_{1}, X_{2}\right]}{\left\langle f_{1}, f_{0}\right\rangle} .
$$

Iterating the above, we obtain $A_{n+1} \cong A_{0}\left[X_{1}, \cdots, X_{n+1}\right] /\left\langle f_{1}, \cdots, f_{n+1}\right\rangle$ and have that $A_{n+1}$ is a finite Galois extension of $A_{n}$ with Galois group $G\left(k_{n+1} / k_{n}\right) ; A_{n+1}$ is also a finite Galois extension of $A_{0}$ with Galois group $G\left(k_{n+1} / d_{0}\right)$.

Now define ideals $I_{n} \leqq B:=A_{0}\left[X_{1}, X_{2}, \cdots\right]$ as follows:

$$
I_{n}:=B\left\langle f_{1}, \cdots, f_{n}\right\rangle+B \cdot\left\langle X_{n+1}, X_{n+2}, \cdots\right\rangle \text { for } n \geqq 1 .
$$

LEMMA 2.3. (1) $I_{n} \geqq I_{n+1}$.

(2) $I_{n} \cap A_{0}=(0)$.

(3) $B / I_{n} \cong A_{n}$.

Proof. (1): Since $f_{n+1} \in A_{0}\left[X_{1}, \cdots, X_{n+1}\right] \subset B$, it follows that 
$B f_{n+1} \subset I_{n}$ so that $I_{n} \geqq I_{n+1}$.

(2): Is clear.

(3): Follows from Lemma (2.2).

Let $U(B)$ have as filter basis the family $\left(I_{n}\right)_{n \geqq 1}$. Then for $I_{n} \geqq$ $I_{n+1}$, we have a commutative diagram

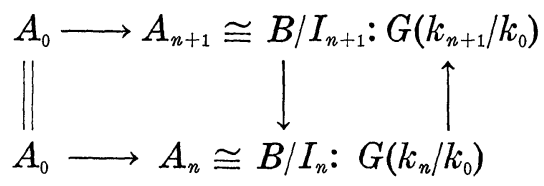

where $A_{i}$ is a Galois extension of $A_{0}$ with group $G\left(k_{2} / k_{0}\right)(i=n, n+1)$. By (1.1) there is a group morphism $G\left(k_{n} / k_{0}\right) \rightarrow G\left(k_{n+1} / k_{0}\right)$ which is injective and satisfies the commutativity criterion of (1.1).

Letting $F=\left(I_{n}\right)_{n \geqq 1}$ and $G: F \rightarrow G r$ be such that $G\left(I_{n}\right)=G\left(k_{n} / k_{0}\right)$ we obtain a quasi-Galois extension $(B, F, G)$ of $A_{0}$.

2. Another quasi-Galois extention. Let $K_{0}<K_{1}<\cdots$ be a tower of Galois field extensions (all finite), $K_{n+1}$ is a finite Galois extension of $K_{n}$, so $K_{n+1} \cong K_{n}\left[X_{n+1}\right] /\left(f_{n+1}\right)$ for a monic polynomial $f_{n+1}$, and repeating the technique of 1 , we get for $A=K_{0}\left[X_{1}, X_{2}, \cdots\right]$ and $F=\left(I_{n}\right)_{n \geqq 1}, I_{n}$ appropriately defined, that $A / I_{n} \cong K_{n}$ so that finally ( $A$, $F, G)$ is a quasi-Galois extension of $K_{0}$ with $G\left(I_{n}\right)=G\left(K_{n} / K_{0}\right)$.

REMARK. In 1 each term $B / I_{n}$ is a local ring, while in 2 each term $A / I_{n}$ is an integral domain. These are two general classes of connected rings. Later we will give an example of a quasi-Galois extension where the approximating terms are not connected, i.e., have proper idempotents.

3. Quasi-Galois extensions in rings of continuous functions. This example is fairly complicated, so $I$ first state the results. Let $\left(X_{i}\right)_{i \in I}$ be a cofiltered family of topological spaces such that $i \leqq j$ in $I$ implies $x_{i j}: X_{i} \rightarrow X_{j}$ is an inclusion for which the identity

$$
x_{i j}^{-i}\left(\operatorname{Top}\left(X_{\imath}\right)\right)=\operatorname{Top}\left(X_{i}\right)
$$

holds. Let $X=\lim _{I} X_{i}$, and let $x_{i}: X_{i} \rightarrow X$ be the colimit morphisms. Then the $x_{2}$ are injective.

Next, let $C:$ Top $\rightarrow R I N$ be the functor assigning to each topological space $X$, the ring of continuous real valued functions with domain $X$, where Top denotes the category of topological spaces.

Lemma 2.4. $C(X) \cong \lim _{I} C\left(X_{i}\right)$ via $f \rightarrow\left(x_{i} f\right)_{i \in I}$.

Now suppose $\left(G_{i}\right)_{i \in I}$ is a cofiltered family of groups such that 
$i \leqq j$ implies $g_{i j}: G_{i} \rightarrow G_{j}$ is the monomorphism, and let $G=\lim _{I} G_{i}$ with $g_{i}: G_{2} \rightarrow G$ being the canonical colimit morphisms. The $\overrightarrow{g_{i}}$ are injective. We will suppose that $G_{i}$ acts continuously on $X_{i}, G_{i}: X_{i} \rightarrow$ $X_{i}$, in such a way that for $i \leqq j$ in $I$ we have a commutative diagram for all $\sigma \in G_{2}$ :

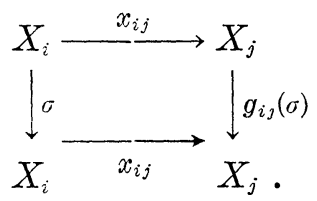

Lemma 2.5. $G$ acts continuously on $X$, and if $g \in G$, there is $I \in$ $I$ for which $g_{i}(\sigma)=g$ and the diagram below is commutative:

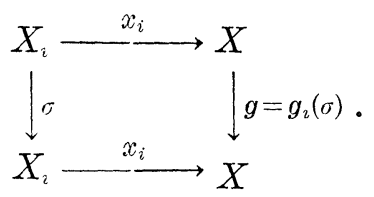

Due to the foregoing assumptions we obtain commutative diagrams:
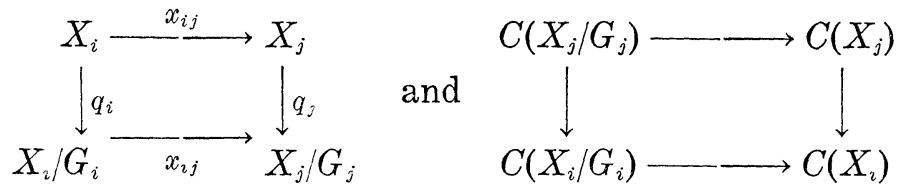

for $i \leqq j$ in $I$, where $X_{i} / G_{\imath}$ is the space of $G_{i}$-orbits of $X_{i}$ with the quotient topology, while $q_{2}$ is the canonical quotient morphism. A more general result than (2.4) is the following:

Lemma 2.6. $C(X / G) \cong \lim _{I} C\left(X_{i} / G_{i}\right)$ via $f \rightarrow\left(f_{i}\right)_{i \in I}$, where $q_{i} f_{i}=$ $x_{2} q f$ and $q: X \rightarrow X / G$ is the quotient map.

Finally, suppose the following conditions are fulfilled.

(a) Each $X_{i}$ is compact.

(b) $G_{i}: X_{i} \rightarrow X_{i}$ is a finite group without fixed points.

(c) Both $C(X) \rightarrow C\left(X_{i}\right)$ and $C(X / G) \rightarrow C\left(X_{i} / G_{i}\right)$ are surjective. Then:

(0) $\operatorname{ker}\left[C(X) \rightarrow C\left(X_{i}\right)\right] \cap C(X / G)=\operatorname{ker}\left[C(X / G) \rightarrow C\left(X_{i} / G_{i}\right)\right]$.

(1) $(C(X), F, H)$ is a quasi-Galois extension of $C(X / G)$, where $F=\left(\operatorname{ker}\left[C(X) \rightarrow C\left(X_{\imath}\right)\right]\right)_{i \in I}$ and $H\left(\operatorname{ker}\left[C(X) \rightarrow C\left(X_{i}\right)\right]\right)=G_{\imath}$.

Proof. Draw the diagram: 


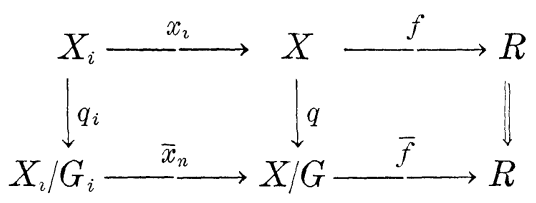

and assume $x_{2} f=0$ and $q \bar{f}=f$. Then $q_{2} \bar{x}_{i} \bar{f}=0$ implies $\bar{x}_{\imath} \vec{f}=0$ which implies $\bar{f} \in \operatorname{ker}\left[C(X / G) \rightarrow C\left(X_{2} / G_{2}\right)\right]$. Conversely, $\bar{x}_{i} \bar{f}=0$ implies $x_{i} q \bar{f}=0$ and $q \bar{f}:=f \in C(X / G) \cap \operatorname{ker}\left[C(X) \rightarrow C\left(X_{i}\right)\right]$ which completes the proof of $(0)$.

For (1), it follows that for each $i \in I$ the diagram

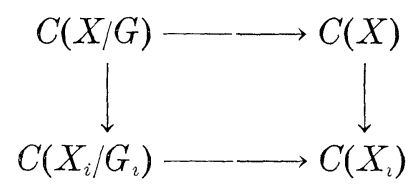

is commutative. $H\left(\operatorname{ker}\left[C(X) \rightarrow C\left(X_{\imath}\right)\right]\right)=G_{\imath}$ acts on $C\left(X_{i}\right)$ by the formula $\sigma f(x)=f(\sigma(x))$ for all $x \in X_{i}$ and $\sigma \in G_{i}$. Since $X_{i}$ is compact and $G_{2}$ acts without fixed points, it follows from (0.2), (2), that $C\left(X_{2}\right) \rightarrow$ $C\left(X_{\imath}\right)$ is a Galois extension with group $G_{\imath}$. Moreover, we have for $i \leqq j$ in $I$, a commutative diagram

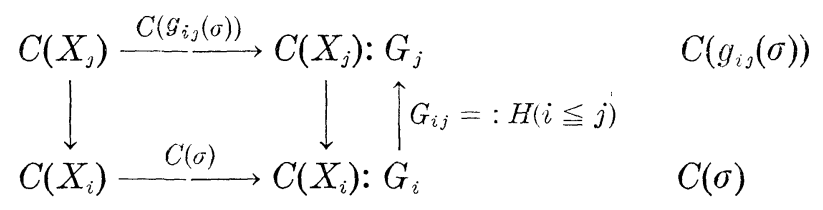

since the corresponding diagram omitting the $C$ 's is commutative.

Letting $U(C(X))$ have as filter basis the family $F=(\operatorname{ker}[C(X) \rightarrow$ $\left.\left.C\left(X_{2}\right)\right]\right)_{i \in I}$ we see that $(C(X), H, F)$ is a quasi-Galois extension of $C(X / G)$.

As example of such a situation as described above, let, for each $n \geqq 1, X_{n}$ be the topological coproduct of $3^{n}$ copies of $[0,1]$, and let $G_{n}$ the cyclic group of order $3^{n}$ acting on $X_{n}$ by permuting the summands. $G_{n}$ acts continuously and has no fixed points, while $X_{n}$ is compact. We have $\lim _{n \geqq 1} G_{n}=Z\left(3^{\infty}\right)$ and $\lim _{n \geqq 1} X_{n}$ is simply the coproduct of a countable $\overrightarrow{n u m b e r}$ of copies of $\overrightarrow{[0}, 1]$, where we interpret always $X_{n} \leqq X_{n+1}$ and $G_{n} \leqq G_{n+1}$. It is clear that the diagrams following (2.4) and (2.5) are commutative, and that the conditions (a)-(c) are fulfilled in this case.

We will now prove assertions (2.4), (2.5), and (2.6).

LEMMA 2.4. $C(X) \cong \lim _{I} C\left(X_{\imath}\right)$.

Proof. For each $i \leqq j$ in $I$, we have by definition a commutative 
diagram:

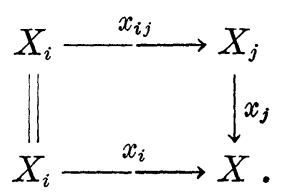

If $\left(f_{i}\right)_{\imath \in I} \in \lim _{\leftarrow} C\left(X_{i}\right)$, then for $i \leqq j$ we have a diagram

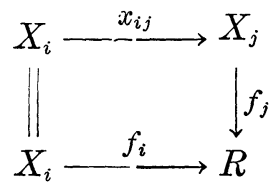

so there is a unique $f: X \rightarrow R$ such that $x_{i} f=f_{i}$ for $i \in I$. This shows that $f \rightarrow\left(x_{i} f\right)_{i \in F}$ is bijective, and the uniqueness guarantees that this mapping is a ring morphism.

LemmA 2.5. $G$ acts continuously on $X$.

Proof. $G$ is formed by taking colimits of diagrams like:

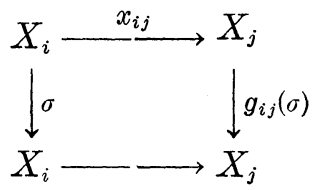

where $j \geqq i$ for all $\sigma \in G(i)$. This leads to commutative diagrams:

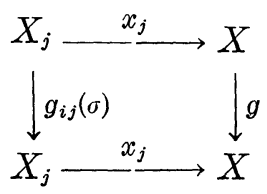

where $g=\lim _{j \geqq i} g_{i j}(\sigma)$. It follows immediately that $x_{j}^{-1} g^{-1}(0) \in \operatorname{Top}\left(X_{j}\right)$ for all $j \geqq \vec{i}$ and all $0 \in \operatorname{Top}(X)$; moreover, if $k \in I$, let $j \geqq i, k$, then $x_{k}^{-1} g^{-1}(0)=x_{k j}^{-1} x_{j}^{-1} g^{-1}(0) \in \operatorname{Top}\left(X_{k}\right)=X_{k j}^{-1}\left(\operatorname{Top}\left(X_{j}\right)\right)$ by definition of $\operatorname{Top}\left(X_{k}\right)$.

Hence, $g$ is continuous.

Lemma 2.6. $C(X / G) \cong \lim _{I} C\left(X_{i} / G_{i}\right)$ via $f \rightarrow\left(\bar{x}_{i} f\right)_{i \in I}$.

Proof. Let $y_{i}: X_{i} / G_{i} \rightarrow Y$ be such that $\bar{x}_{i j} y_{j}=y_{i}$ for $i \leqq j$ in $I$. Then composing $q_{i}: X_{i} \rightarrow X_{i} / G_{i}$ with $y_{i}$ yields a family $\left(q_{i} y_{i}\right)_{i \in I}$ compatible with the $x_{i j}: X_{i} \rightarrow X_{j}$ for $i \leqq j$. Hence, there is a unique $y: X \rightarrow Y$ 
such that $x_{i} y=q_{i} y_{i}$ for $i \in I$ by (2.4). Next, let $g \in G$, say $g=g_{i}(\sigma)$ for $\sigma \in G(i)$. We then have the equations: $x_{j} g y=g_{i j}(\sigma) x_{j} y=x_{j} y$ since $y$ is constant on $G_{j}$-orbits of $X_{j}$, i.e., $x_{j} y=q_{j} y_{j}$. Passing to the colimit over $j \geqq i$, we get $g y=y$ showing that $y$ is constant on $G$-orbits of $X$. Hence, there is a unique $\bar{y}: X / G \rightarrow Y$ such that $y=q \bar{y}$. Since $q_{i}$ is surjective and $q_{i} y_{i}=x_{\imath} y=x_{\imath} q \bar{y}=q_{\imath} \bar{x} \bar{y}$, we conclude that $y_{\imath}=$ $\bar{x}_{i} \bar{y}$ for all $i \in I$. Thus, the mapping $f \rightarrow\left(\bar{x}_{i} f\right)_{i \in I}$ is bijective and as before the uniqueness assures that it is a ring morphism.

4. A non-connected quasi-Galois extension. Let $(A, F, G)$ be a quasi-Galois extension of $k$ and let $n \geqq 2$. Put $A^{n}=A \pi \cdots \pi A$ ( $n$ factors) and $F^{(n)}=\left\{I^{n} \mid I \in F\right\}$. The diagonal map $\Delta: k \rightarrow A^{n}$ makes $A$ a $k$-algebra, and $I \in F$ implies $A^{n} / I^{n} \cong(A / I)^{n}$. Moreover, $I \leqq I^{\prime}$ in $F$ induces $\left(a_{I^{\prime}}^{I}\right):(A / I)^{n} \rightarrow\left(A / I^{\prime}\right)^{n}$ which is surjective. It follows from [2] (Chapter IX $\S 7$, Prop. 7.3) by induction that $(A / I)^{n}$ is a separable $k_{I}$-algebra via the diagonal map $\Delta_{I}: k_{I} \rightarrow(A / I)^{n}$, where $k_{I}=k / k \cap I$.

Next, let $G^{n}(I)=G(I) \pi \cdots \pi G(I)$ (n factors) and let $H(I)$ denote the diagonal subgroup of $G^{n}(I)$, that is the image of the diagonal map $\Delta: G(I) \rightarrow G^{n}(I) . \quad G^{n}(I)$ acts componentwise on $(A / I)^{n}$. Let $H$ be any subgroup of the symmetric group of $n$ letters which moves all the letters to all positions, e.g., the cyclic group of order $n$. We think of $H$ as acting on each $(A / I)^{n}$ as a permutation of the factors. Finally, let $K(I)$ be the normal product of $H$ with $H(I)$, so that each element of $K(I)$ may be put in the form $\pi \Delta(\sigma)$ with $\pi \in H$ and $\sigma \in$ $H(I)$.

Lemma 2.7. (a) $K(I)$ acts on $(A / I)^{n}$ with fixed ring $\Delta_{I}(k / k \cap I)$ for $I \in F$.

(b) $(A / I)^{n}$ is a Galois extension of $k / k \cap I$ with group $K(I)$ for $I \in F$.

Proof. It is clear how $K(I)$ acts on $(A / I)^{n}$ using the representation of elements of $K(I)$ in the form $\pi \Delta(\sigma)$. If $\left(a_{1}, \cdots, a_{n}\right)$ is fixed by $K(I)$, then because $K(I)$ moves each component to every other component, and each component lies in $k / k \cap I \cdot 1$, we must have that the element $\left(a_{1}, \cdots, a_{n}\right) \in \Delta_{I}(k / k \cap I)$, proving (a).

Next, let $\left(x_{2}\right),\left(y_{2}\right)$ be two families of elements of $A / I$ such that $\sum_{i} x_{i} \sigma\left(y_{2}\right)=\delta_{1 \sigma}$ for all $\sigma \in G(I)$. Such exist by (0.2), (1). Then we have $\sum_{i} \Delta_{I}\left(x_{i}\right) \pi \Delta(\sigma)\left(\Delta_{I}\left(y_{i}\right)\right)=\Delta_{I}\left(\sum_{i} x_{\imath} \sigma\left(y_{i}\right)\right)=\Delta_{I}\left(\hat{o}_{1 \sigma}\right)=\delta_{1\lrcorner(\sigma)}=\delta_{1 \pi \Delta(\sigma)} ;$ hence, $(\mathrm{b})$ holds using (0.2), (1), again.

There is an evident group morphism $K\left(I^{\prime}\right) \rightarrow K(I)$ extending $G\left(I^{\prime}\right) \rightarrow$ $G(I)$ which is monic. We denote the so generated functor by $K: F^{(n)} \rightarrow$ $G$, and obtain a quasi-Galois extension $\left(A^{n}, F^{(n)}, K\right)$ of $k$ such that $(A / I)^{n}$ is not connected. 


\section{REFERENCES}

1. Friehorst Ballier, Über linear topologische Algebren, J. Reine Angew. Math., 195 (1955-56), 42-75.

2. H. Cartan and S. Eilenberg, Homological Algebra, Princeton Univ. Press, Princeton, N. J., 1956.

3. S. U. Chase, D. K. Harrison, and A. Rosenberg, Galois Theory and Galois Cohomology of Commutative Rings, Memoirs, Amer. Math. Soc., no. 52, 1965.

4. F. DeMeyer and E. Ingraham, Separable Algebras Over Commutative Rings, Lect. Notes in Math., v. 181, Springer-Verlag-Belin-New York, 1971.

5. J. Dieudonne, Linearly compact spaces and double vector spaces over s fields, Amer.

J. Math., 73 (1951), 13-19.

6. B. L. Elkins, A ramification theory for linear topological rings, (to appear).

7. H. Röhrl, Class notes 1966. University of California, San Diego.

8. J. P. Serre, Corps Locaux, Actualite, Sci. et. Ind., no. 1296, Hermann, Paris, 1962.

Received November 22, 1972. These results were obtained in the author's 1968 $\mathrm{Ph}$. D. thesis written under the direction of Professor Helmut Röhrl at UCSD, La Jolla, California.

OHIo State UNIVERSity 



\section{PACIFIC JOURNAL OF MATHEMATICS}

\section{EDITORS}

RICHARD ARENS (Managing Editor)

University of California

Los Angeles, California 90024

R. A. BeAumont

University of Washington

Seattle, Washington 98105
J. DugundJI*

Department of Mathematics

University of Southern California

Los Angeles, California 90007

D. Gilbarg and J. Milgram

Stanford University

Stanford, California 94305

\section{ASSOCIATE EDITORS}

E. F. BECKENBACH

B. H. NEUMANN

F. WOLF

K. YoSHIDA

\section{SUPPORTING INSTITUTIONS}

UNIVERSITY OF BRITISH COLUMBIA
CALIFORNIA INSTITUTE OF TECHNOLOGY
UNIVERSITY OF CALIFORNIA
MONTANA STATE UNIVERSITY
UNIVERSITY OF NEVADA
NEW MEXICO STATE UNIVERSITY
OREGON STATE UNIVERSITY
UNIVERSITY OF OREGON
OSAKA UNIVERSITY

UNIVERSITY OF BRITISH COLUMBIA CALIFORNIA INSTITUTE OF TECHNOLOGY UNIVERSITY OF CALIFORNIA MONTANA STATE UNIVERSITY NEW MEXICO STATE UNIVERSITY UNIVERSITY OF OREGON OSAKA UNIVERSITY
UNIVERSITY OF SOUTHERN CALIFORNIA STANFORD UNIVERSITY UNIVERSITY OF TOKYO UNIVERSITY OF UTAH WASHINGTON STATE UNIVERSITY UNIVERSITY OF WASHINGTON AMERICAN MATHEMATICAL SOCIETY NAVAL WEAPONS CENTER

* C. R. DePrima California Institute of Technology, Pasadena, CA 91109, will replace J. Dugundji until August 1974. 


\section{Pacific Journal of Mathematics}

\section{Vol. 51, No. $1 \quad$ November, 1974}

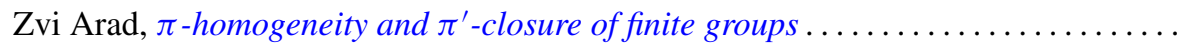

Ivan Baggs, A connected Hausdorff space which is not contained in a maximal

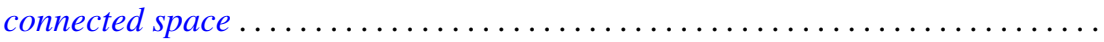

Eric Bedford, The Dirichlet problem for some overdetermined systems on the unit ball in $C^{n}$

R. H. Bing, Woodrow Wilson Bledsoe and R. Daniel Mauldin, Sets generated by

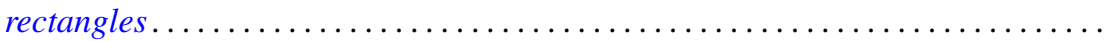

Carlo Cecchini and Alessandro Figà-Talamanca, Projections of uniqueness for

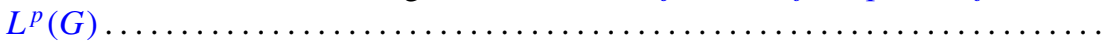

Gokulananda Das and Ram N. Mohapatra, The non absolute Nörlund summability of Fourier series .

Frank Rimi DeMeyer, On separable polynomials over a commutative ring ........ Richard Detmer, Sets which are tame in arcs in $E^{3} \ldots \ldots \ldots \ldots \ldots \ldots \ldots \ldots$

William Erb Dietrich, Ideals in convolution algebras on Abelian groups ..........

Bryce L. Elkins, A Galois theory for linear topological rings .................

William Alan Feldman, A characterization of the topology of compact convergence on $C(X)$.

Hillel Halkin Gershenson, A problem in compact Lie groups and framed cobordism

Samuel R. Gordon, Associators in simple algebras.

Marvin J. Greenberg, Strictly local solutions of Diophantine equations

Jon Craig Helton, Product integrals and inverses in normed rings . . . . . . . . . . . .

Domingo Antonio Herrero, Inner functions under uniform topology . . .

Jerry Alan Johnson, Lipschitz spaces .

Marvin Stanford Keener, Oscillatory solutions and multi-point boundary value

functions for certain nth-order linear ordinary differential equations.

John Cronan Kieffer, A simple proof of the Moy-Perez generalization of the

Shannon-McMillan theorem .......................

Joong Ho Kim, Power invariant rings

Gangaram S. Ladde and V. Lakshmikantham, On flow-invariant sets .

Roger T. Lewis, Oscillation and nonoscillation criteria for some self-adjoint even

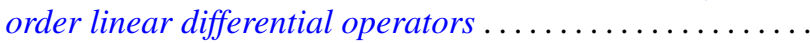

Jürg Thomas Marti, On the existence of support points of solid convex sets ..

John Rowlay Martin, Determining knot types from diagrams of knots . .

James Jerome Metzger, Local ideals in a topological algebra of entire functions

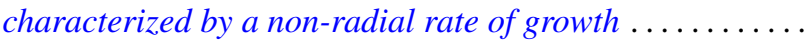

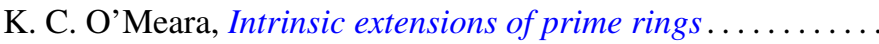

Stanley Poreda, A note on the continuity of best polynomial approximations ..

Robert John Sacker, Asymptotic approach to periodic orbits and local prolongations

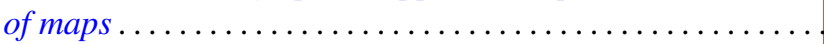

Eric Peter Smith, The Garabedian function of an arbitrary compact set . .

Arne Stray, Pointwise bounded approximation by functions satisfying a side condition

John St. Clair Werth, Jr., Maximal pure subgroups of torsion complete abelian

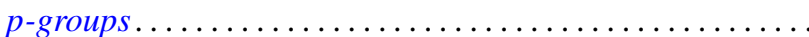

